\title{
Likelihood of acute coronary syndrome in emergency department chest pain patients varies with time of presentation
}

\author{
Ulf Ekelund ${ }^{1 *}$, Mahin Akbarzadeh' ${ }^{1}$, Ardavan Khoshnood ${ }^{1}$, Jonas Björk ${ }^{2}$ and Mattias Ohlsson ${ }^{3}$
}

\begin{abstract}
Background: There is a circadian and circaseptal (weekly) variation in the onset of acute coronary syndrome (ACS). The aim of this study was to elucidate whether the likelihood of ACS among emergency department (ED) chest pain patients varies with the time of presentation.

Methods: All patients presenting to the Lund ED at Skåne University Hospital with chest pain or discomfort during 2006 and 2007 were retrospectively included. Age, sex, arrival time at the ED and discharge diagnose (ACS or not) were obtained from the electronic medical records.

Results: There was a clear but moderate circadian variation in the likelihood of ACS among presenting chest pain patients, the likelihood between 8 and 10 am being almost twice as high as between 6 and 8 pm. This was mainly explained by a variation in the ACS likelihood in females and patients under 65 years, with no significant variation in males and patients over 65 years. There was no significant circaseptal variation in the ACS likelihood.

Conclusions: Our results indicate that there is a circadian variation in the likelihood of ACS among ED chest pain patients, and suggest that physicians should consider the time of presentation to the ED when determining the likelihood of ACS.
\end{abstract}

\section{Background}

Despite tremendous progress in the treatment of acute coronary syndrome (ACS; i.e. unstable angina, UA, or acute myocardial infarction, AMI), our ability to diagnose or rule out ACS in emergency department (ED) chest pain patients remains relatively poor. "Rule-out" admissions to inhospital care are common, and some 7 of 10 patients admitted with a suspicion of ACS prove not to have it $[1,2]$. Because of the diagnostic uncertainty, ED management of patients with suspected ACS depends on the likelihood of ACS. When assessing the ACS likelihood, the ED physician considers a number of diagnostic factors, often with a Bayesian approach [3] using the likelihood ratio (LR) for each test [4-7]. For instance, the positive LR of a new ST elevation on the ECG has been reported to be 5.7-53.9, a new ST depression 3.0-5.2, pain radiating to both arms or shoulders

\footnotetext{
* Correspondence: ulf.ekelund@med.lu.se

'Department of Emergency Medicine, Skåne University Hospital, Lund SE-221 85, Sweden

Full list of author information is available at the end of the article
}

4.1, to the left arm 2.3, excertional chest pain 2.5, a history of myocardial infarction 1.5-3.0, diaphoresis 2.0, chest pain as the most important symptom 2.0, nausea or vomiting 1.9, positional chest pain 0.3 and pleuritic chest pain 0.2 [4-7]. To improve management, new diagnostic methods and blood tests have been suggested [8], as well as a large number of decision support models to predict the likelihood of ACS, e.g. [9-12]. In general however, routine evaluation of ED chest pain patients has not improved in later years.

It is well known that there are circadian, circaseptal (weekly) and seasonal variations in the onset of ACS [13-17], and even a circadian variation in AMI size [18]. A circadian variation of the ACS probability in chest pain patients arriving at the ED may therefore be present but has not been demonstrated. Accordingly, there is currently no basis for ED physicians to use the time of presentation to the ED as a factor when determining the likelihood of ACS, and no decision support model so far includes the time of presentation.

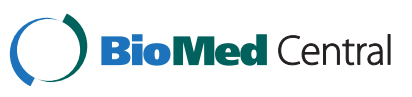

(C) 2012 Ekelund et al.; licensee BioMed Central Ltd. This is an Open Access article distributed under the terms of the Creative Commons Attribution License (http://creativecommons.org/licenses/by/2.0), which permits unrestricted use, distribution, and reproduction in any medium, provided the original work is properly cited. 
The aim of this study was to explore the impact of the time of arrival at the ED and the weekday on the likelihood of ACS in ED chest pain patients.

\section{Methods \\ Setting}

The Lund ED at Skåne University Hospital has a catchment area of some 300,000 inhabitants and receives a total of 65,000 patients per year with problems related to Internal Medicine, Neurology, Surgery, Orthopedics and Urology. In the hospital, there is a cardiac intensive care unit with 19 beds and an intermediate care ward with ECG monitoring for cardiac patients at 19 beds. Percutaneous coronary intervention and coronary bypass surgery are available 24 hours/day. During the patient inclusion period, there was no systematic diagnostic protocol for patients with suspected ACS, and no dedicated chest pain unit.

\section{Patients and diagnoses}

We retrospectively included all patients presenting with non-traumatic chest pain or discomfort as chief complaint, as identified by the triage nurse and/or the responsible physician, at the Lund ED from January $1^{\text {st }}$ 2006 to December $31^{\text {st }} 2007$. Data for each patient regarding age and sex, arrival time at the ED, admission to inhospital care or not, and discharge diagnosis (ACS or not) were retrieved from the electronic patient records.

During the study period, diagnostic criteria for ACS (acute myocardial infarction or unstable angina pectoris) at the hospital were those recommended by the European Society of Cardiology, the American College of Cardiology and the American Heart Association [19-21]. Discharge diagnoses were made by the attending physician at the ED, or, after hospital admission, by the responsible specialist physician at the ward. No follow-up of the patients sent home from the ED was performed. The data used in the analysis were those used in the actual clinical care of the patients, and they are therefore likely to represent the routine care situation.

\section{Statistical analysis}

We used SPSS for Windows version 18 (IBM Corp.) for the statistical analyses. The circadian and circaseptal variation in the probability of ACS was modeled using multiple logistic regression with time (12 different groups) and weekday as categorical independent variables. Sex and age (9 groups) were also included in the regression models to control for potential differences in sex and age distribution in the patient inflow at different times of the day and at different days of the week. The analyses of circadian and circaseptal variation were conducted for the entire data set, but also stratified for sex and for age (below/at least 65 years old).

\section{Ethical approval}

This study was approved (dnr 384/2007) by the Regional Ethics Committee at Lund.

\section{Results}

As can be seen in Figure 1, 11219 ED patient visits with acute chest pain or discomfort were identified during 2006 and 2007. Seventy-eight visits were excluded due to age $<18$ years or missing data, leaving 11141 visits by 8763 patients as the study sample. Figure 2 depicts the age distribution for all patient visits and for those admitted to in-hospital care. In Table 1, the ACS likelihoods for the studied patient subgroups are shown. Among the ACS cases, $35 \%$ were women and $67 \%$ were $\geq 65$ years.

\section{Patients presenting to the ED}

As can be seen in Figure 3, the peak ED inflow of chest pain patients was between 10 am and noon. This pattern was similar for men and women, and for patients under and over 65 years. The inflow of ACS patients showed a similar pattern but was highest already at 9 to 10 am (data not shown). The likelihood of ACS among presenting chest pain patients showed a clear but moderate circadian variation (Figure $4 ; \mathrm{p}=0.01$ in the multiple logistic regression model), with a peak 8-10 am (10.6\%) and a nadir at 6-8 pm (5.6\%). This diurnal variation was mainly explained by a variation in the ACS likelihood for female $(\mathrm{p}=0.006)$ and younger patients $(<65$ years; $\mathrm{p}=0.02)$, with no significant variation in male $(\mathrm{p}=0.07)$ and older patients $(\mathrm{p}=0.26)$. In women, the highest and lowest likelihoods were $9 \%$ and $2.5 \%$.

The circaseptal variation of chest pain patient inflow was modest with Monday as the busiest day (18\% of weekly patients) and Friday as the least busy day (12\%). There was no significant circaseptal variation in the likelihood of ACS ( $>>0.3)$.

\section{Patients admitted to in-hospital care}

Among chest pain patients admitted from the ED to inhospital care $(n=5233)$, the average ACS likelihood was higher for men than for women, and also higher for older than for younger patients (Table 1). The likelihood of ACS among admitted cases was $20 \%$ for those arriving at the ED at 8-10 am, and $13 \%$ at $6-8 \mathrm{pm}$, but the circadian variation was not statistically significant $(p=0.06)$.

\section{Discussion}

This study indicates that there is a diurnal variation in the likelihood of ACS among chest pain patients presenting at the ED. To our knowledge, this variation has not been described before. The diurnal variation was more marked among females and younger patients, and was not explained by differences in sex and age distribution in the patient inflow at different times of the day. 


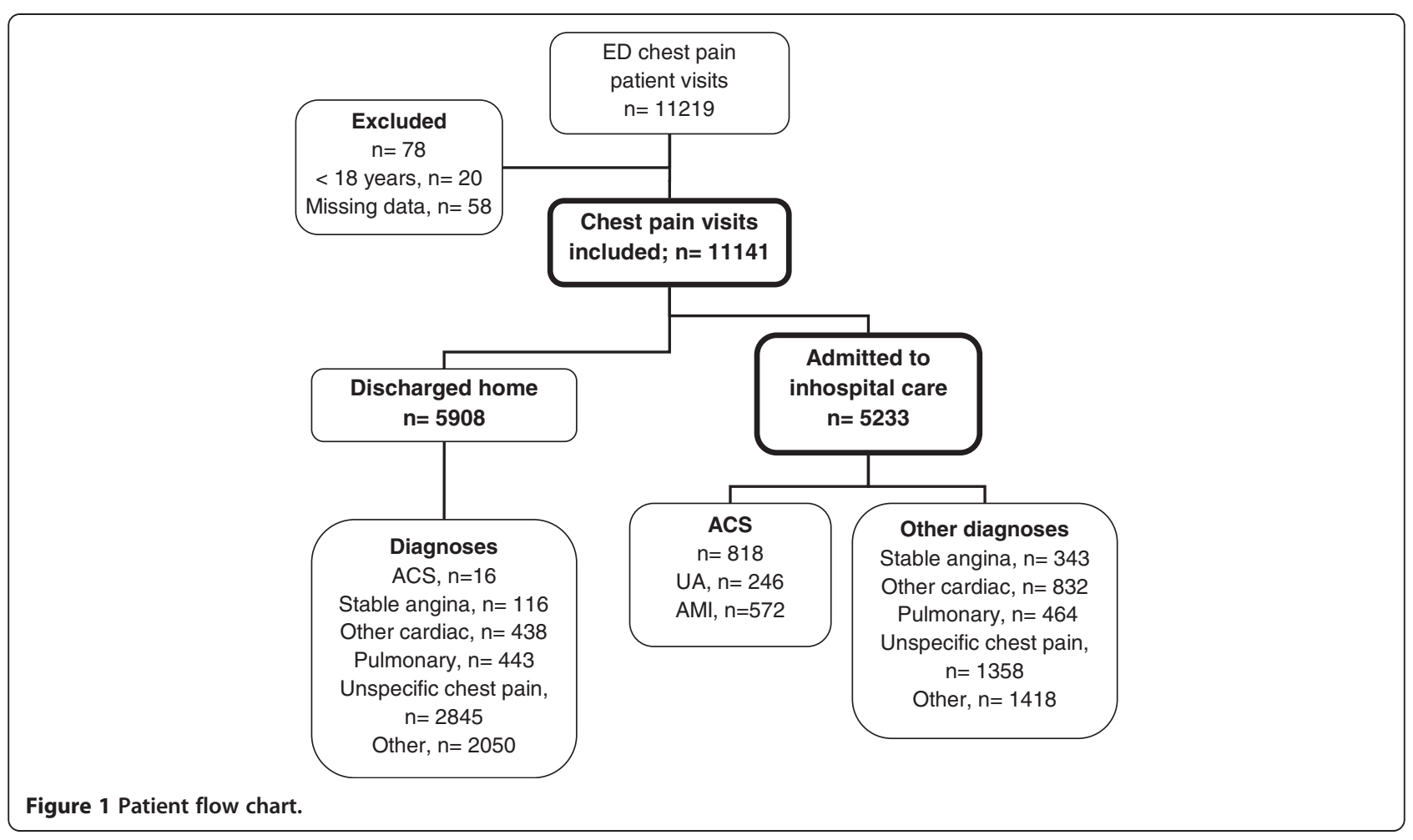

The circadian variation in the ACS likelihood was clear but moderate, with a higher likelihood midnight-10 am (Figure 4), a peak at 6-10 am and a nadir at 6-8 pm. The obvious reason for this likelihood variation was the different diurnal inflows of chest pain patients with and without ACS. The causes of the different inflows of these groups are unclear, but there are at least two possible explanations. The first is the well established circadian variation of ACS symptom onset [13-17], which would

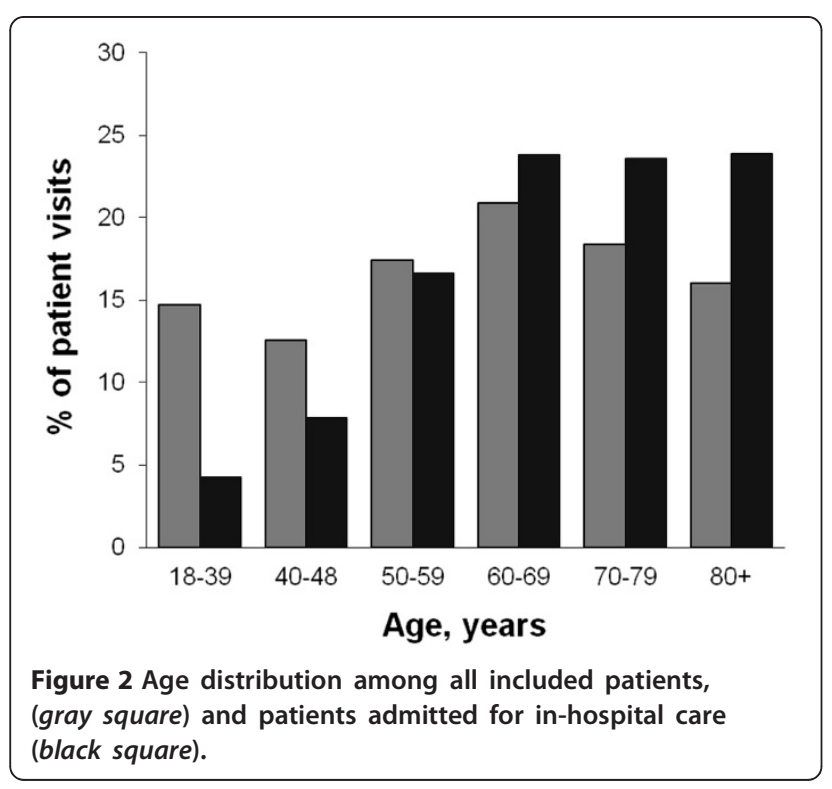

cause ACS patients to present at the ED primarily in the morning. The second is that patients with milder chest pain and "harmless" diagnoses may hesitate to go to the ED during the inconvenient hours between midnight and $10 \mathrm{am}$. Other possible causes include different prehospital delays in the two groups owing to different symptom histories, comorbidities and/or availabilities of transportation (e.g. ambulance vs public transportation). Whatever the cause, the present data indicate that time of presentation to the ED is a diagnostic factor when determining the likelihood of ACS in ED chest pain patients.

The ED physician often determines the ACS likelihood using Bayesian reasoning [3] and diagnostic information mainly from the symptom history, the ECG and blood markers of myocardial injury, together with factors such as age, sex and previous diseases [4-7,20]. In the present study, the odds ratio for ACS for a patient presenting 8-10 am as compared to $6-8 \mathrm{pm}$ was 2.0. This odds ratio is in the same order of magnitude as the impact on the odds (i.e. LR) for ACS of pain radiating to the left arm (LR 2.3), diaphoresis (LR 2.0), a history of myocardial infarction (LR 1.5-3.0), and chest pain as the most important symptom (LR 2.0), and larger than the impact of male sex in the present study (odds ratio 1.6; see Table 1). Time of presentation to the ED may thus be as important as many well-established diagnostic factors when assessing the likelihood of ACS.

The demonstration of a diurnal variation of the ACS likelihood may have at least two clinical implications. 
Table 1 Number, age and ACS likelihood among presenting and admitted study cases

\begin{tabular}{lccccc}
\hline & $\begin{array}{c}\text { Mean age } \\
\text { years (SD }\end{array}$ & ED visits $(\mathbf{n})$ & $\begin{array}{c}\text { Admitted } \\
\text { cases }(\mathbf{n})\end{array}$ & \begin{tabular}{c} 
ACS likelihood (prevalence) \\
\cline { 4 - 6 } Cases presenting to the ED
\end{tabular} & $\begin{array}{c}\text { Admitted cases } \\
\text { All patient visits }\end{array}$ \\
Men & $60(18)$ & 11141 & 5233 & $9 \%$ & $16 \%$ \\
Women & $59(18)$ & 5789 & 2979 & $6 \%$ & $18 \%$ \\
Patients $<65$ years & $62(19)$ & 5352 & 2254 & $4.5 \%$ & $13 \%$ \\
Patients $\geq 65$ years & $47(13)$ & 6263 & 2152 & $11 \%$ & $13 \%$ \\
\hline
\end{tabular}

${ }^{\mathrm{a} S t a n d a r d}$ deviation.

First, knowledge of this variation might influence the ED physician's decision-making. Patients presenting at 9 am had almost twice the ACS risk of those presenting at $7 \mathrm{pm}$ (Figure 4), and in female patients, the risk was 3 times higher. Since the likelihood variation must still be viewed as modest however, clinicians may take the time of arrival into account mainly when ECG, blood markers and symptoms are inconclusive, like they do with other clinical information with a modest LR such as gender, diaphoresis or a previous MI [5,7]. Second, mathematical decision support models for the prediction of ACS may be improved by the addition of a factor related to the time of presentation. Published models so far have included e.g. the ECG, previous coronary artery disease, patient age, pain duration, localization and similarity to previous angina or AMI, diabetes and sex [10-12,22,23]. Currently, these decision support models are rarely used in routine care, but if significantly improved by a time factor, their clinical use may increase.

The present inflow pattern of chest pain patients was very similar to the overall patient inflow at our and other [24-26] EDs, with a noon peak and an early morning nadir. Further, the inflow pattern of ACS patients was similar to that reported for AMI patients in the UK [27].

In patients with acute myocardial infarction (AMI), the average delay from symptom onset to presentation at the hospital is 2-4 $\mathrm{h}$ [28]. If the delay was similar for the ACS patients in the present study, the incidence of ACS

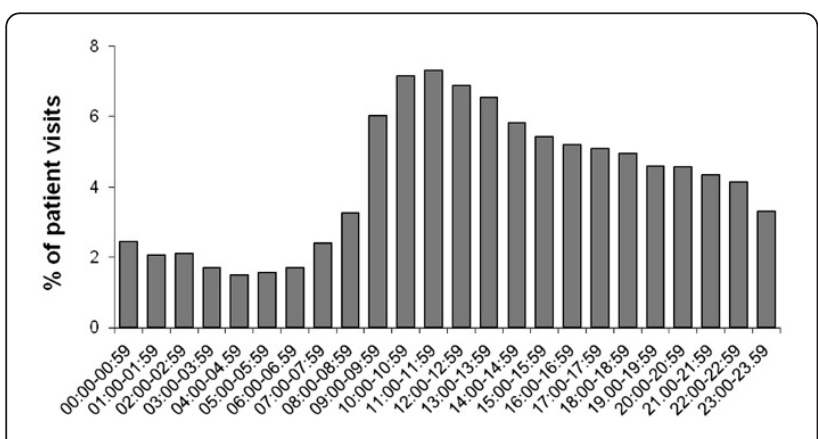

Figure 3 Circadian inflow of chest pain patients to the ED. symptom onset was highest 6-8 am. This is somewhat earlier than in previous studies, where the peak incidence of AMI symptom onset was at 8-10 am [17,29-31]. However, the average delay may be different depending on society resources, health care organization and patient population [28], and may well be different in the present study which included ACS cases than in previous studies with only AMI cases.

\section{Study limitations}

This study was performed in a single university hospital ED and did not include data on chest pain duration or characteristics, prehospital delay or comorbidities in the patients. Although the diurnal presentation pattern and the age and sex distribution suggest that our patients were similar to those in other EDs [24-27], the present results need to be confirmed in other patient populations and health care settings. No follow-up was performed of the patients sent home from the ED, and a small fraction of the ACS cases might therefore have been missed. Since this is probably rare at our ED [32], it is unlikely that this would have influenced the results significantly.

\section{Conclusions}

These results indicate that there is a circadian variation in the likelihood of ACS among chest pain patients presenting to the ED. The causes of the variation are likely multiple, and probably include the well-known diurnal

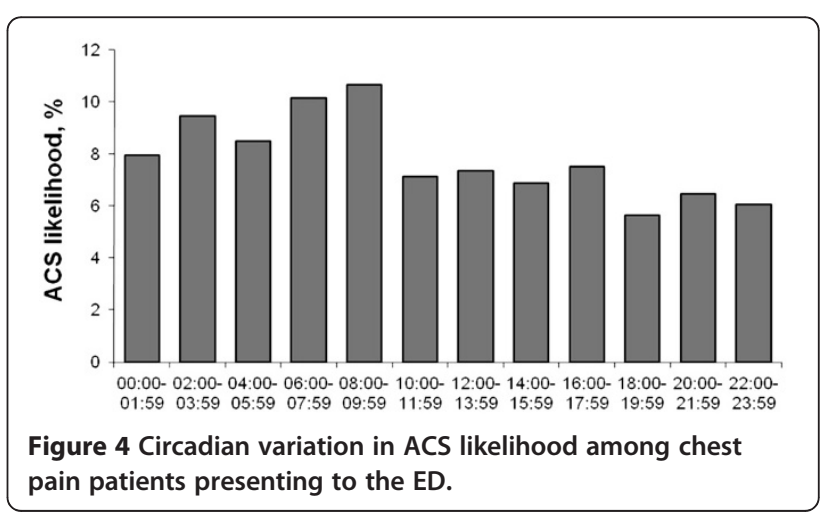


variation in ACS symptom onset. The results suggest that physicians should consider the time of arrival at the ED when assessing the likelihood of ACS in ED chest pain patients, but additional studies are needed to confirm this in other populations and health care settings.

\section{Competing interests}

The authors declare that they have no competing interests.

\section{Authors' contributions}

UE conceived and designed the study, interpreted the data and wrote the manuscript. MA and AK collected and interpreted the data and critically revised the manuscript. JB provided expert statistical advice in the design and presentation in the study and critically revised the manuscript. MO collected and organized the electronic data, and critically revised the manuscript. All authors read and approved the final manuscript.

\section{Acknowledgements}

This study was funded by the Region Skåne.

\section{Sources of funding}

This work was supported by an ALF grant at Skåne University Hospital, Lund, Sweden, by the Region Skåne, The Swedish Heart and Lung foundation and the Laerdal foundation for Acute Medicine.

\section{Author details}

'Department of Emergency Medicine, Skåne University Hospital, Lund SE-221 85, Sweden. ${ }^{2}$ Region Skåne competence centre for clinical research, Lund, Sweden. ${ }^{3}$ Department of Theoretical Physics, Lund University, Lund, Sweden.

Received: 12 April 2012 Accepted: 30 April 2012

Published: 8 August 2012

\section{References}

1. Pope JH, Ruthazer R, Beshansky JR, Griffith JL, Selker HP: Clinical features of Emergency Department Patients presenting with symptoms suggestive of acute cardiac ischemia: a multicenter study. J Thromb Thrombolysis 1998, 6(1):63-74

2. Ekelund U, Nilsson HJ, Frigyesi A, Torffvit O: Patients with suspected acute coronary syndrome in a university hospital emergency department: an observational study. BMC Emerg Med 2002, 2(1):1-7.

3. Grant DC, Keim SM, Telfer J: Teaching Bayesian analysis to Emergency Medicine residents. J Emerg Med 2006, 31(4):437-440.

4. Goodacre S, Locker T, Morris F, Campbell S: How useful are clinical features in the diagnosis of acute, undifferentiated chest pain? Acad Emerg Med 2002, 9(3):203-208.

5. Panju AA, Hemmelgarn BR, Guyatt GH, Simel DL: The rational clinical examination. Is this patient having a myocardial infarction? JAMA 1998, 280(14):1256-1263.

6. Bruyninckx R, Aertgeerts B, Bruyninckx P, Buntinx F: Signs and symptoms in diagnosing acute myocardial infarction and acute coronary syndrome: a diagnostic meta-analysis. Br J Gen Pract 2008, 58(547):105-111.

7. Swap CJ, Nagurney JT: Value and limitations of chest pain history in the evaluation of patients with suspected acute coronary syndromes. Jama 2005, 294(20):2623-2629.

8. Ekelund U, Forberg JL: New methods for improved evaluation of patients with suspected acute coronary syndrome in the emergency department. Emerg Med J 2007, 24(12):811-814.

9. Tierney WM, Roth BJ, Psaty B, McHenry R, Fitzgerald J, Stump DL, Anderson FK, Ryder KW, MCDonald CJ, Smith DM: Predictors of myocardial infarction in emergency room patients. Crit Care Med 1985, 13(7):526-531.

10. Selker HP, Beshansky JR, Griffith JL, Aufderheide TP, Ballin DS, Bernard SA, Crespo SG, Feldman JA, Fish SS, Gibler WB, et al: Use of the acute cardiac ischemia time-insensitive predictive instrument (ACI-TIPI) to assist with triage of patients with chest pain or other symptoms suggestive of acute cardiac ischemia. A multicenter, controlled clinical trial. Ann Intern Med 1998, 129(11):845-855.
11. Harrison RF, Kennedy RL: Artificial neural network models for prediction of acute coronary syndromes using clinical data from the time of presentation. Ann Emerg Med 2005, 46(5):431-439.

12. Goldman L, Cook EF, Brand DA, Lee TH, Rouan GW, Weisberg MC, Acampora D, Stasiulewicz C, Walshon J, Terranova G: A computer protocol to predict myocardial infarction in emergency department patients with chest pain. N Engl J Med 1988, 318(13):797-803.

13. Hammoudeh AJ, Alhaddad IA: Triggers and the onset of acute myocardial infarction. Cardiol Rev 2009, 17(6):270-274.

14. Bhalla A, Sachdev A, Lehl SS, Singh R, D'Cruz S: Ageing and circadian variation in cardiovascular events. Singapore Med J 2006, 47(4):305-308

15. Mickley $H$, Pless $P$, Nielsen JR, Moller M: Circadian variation of transient myocardial ischemia in the early out-of-hospital period after first acute myocardial infarction. Am J Cardiol 1991, 67(11):927-932.

16. Rana JS, Mukamal KJ, Morgan JP, Muller JE, Mittleman MA: Circadian variation in the onset of myocardial infarction: effect of duration of diabetes. Diabetes 2003, 52(6):1464-1468.

17. ISIS-2 (Second International Study of Infarct Survival) Collaborative Group: Morning peak in the incidence of myocardial infarction: experience in the ISIS-2 trial. Eur Heart J 1992, 13(5):594-598.

18. Suarez-Barrientos A, Lopez-Romero P, Vivas D, Castro-Ferreira F, Nunez-Gil I, Franco E, Ruiz-Mateos B, Garcia-Rubira JC, Fernandez-Ortiz A, Macaya C, et al: Circadian variations of infarct size in acute myocardial infarction. Heart 2011, 97(12):970-976.

19. Myocardial infarction redefined--a consensus document of The Joint European Society of Cardiology/American College of Cardiology Committee for the redefinition of myocardial infarction. Eur Heart J 2000, 21(18):1502-1513.

20. Braunwald E, Antman EM, Beasley JW, Califf RM, Cheitlin MD, Hochman JS, Jones RH, Kereiakes D, Kupersmith J, Levin TN, et al: ACC/AHA 2002 guideline update for the management of patients with unstable angina and non-ST-segment elevation myocardial infarction-summary article. J Am Coll Cardiol 2002, 40(7):1366-1374.

21. Bertrand ME, Simoons ML, Fox KA, Wallentin LC, Hamm CW, McFadden E, De Feyter PJ, Specchia G, Ruzyllo W: Management of acute coronary syndromes in patients presenting without persistent ST-segment elevation. Eur Heart J 2002, 23(23):1809-1840.

22. Pozen MW, D'Agostino RB, Selker HP, Sytkowski PA, Hood WB Jr: A predictive instrument to improve coronary-care-unit admission practices in acute ischemic heart disease. A prospective multicenter clinical trial. N Engl J Med 1984, 310(20):1273-1278.

23. Lopez de Sa E, Lopez-Sendon J, Anguera I, Bethencourt A, Bosch X: Prognostic value of clinical variables at presentation in patients with non-ST-segment elevation acute coronary syndromes: results of the Proyecto de Estudio del Pronostico de la Angina (PEPA). Medicine 2002, 81(6):434-442. Baltimore.

24. Ekelund U, Kurland L, Eklund F, Torkki P, Letterstal A, Lindmarker P, Castren $M$ : Patient throughput times and inflow patterns in Swedish emergency departments. A basis for ANSWER, A National SWedish Emergency Registry. Scand I Trauma Resusc Emerg Med 2011, 19:37.

25. Downing A, Wilson R: Temporal and demographic variations in attendance at accident and emergency departments. Emerg Med J 2002, 19(6):531-535.

26. Pitts SR, Niska RW, Xu J, Burt CW: National Hospital Ambulatory Medical Care Survey: 2006 Emergency Department Summary. In: National Health Statistics Reports. Washington DC: US Department of health and human services; 2008:1-14.

27. Accident and Emergency Attendances in England 2009-10. In.: National Health Service. UK: The Health and Social Care Information Centre; 2011:1-55.

28. Nguyen HL, Saczynski JS, Gore JM, Goldberg RJ: Age and sex differences in duration of prehospital delay in patients with acute myocardial infarction: a systematic review. Circ Cardiovasc Qual Outcomes 2010, 3(1):82-92.

29. Hjalmarson A, Gilpin EA, Nicod P, Dittrich H, Henning H, Engler R, Blacky AR, Smith SC Jr, Ricou F, Ross J Jr: Differing circadian patterns of symptom onset in subgroups of patients with acute myocardial infarction. Circulation 1989, 80(2):267-275.

30. Leiza JR, de Llano JM, Messa JB, Lopez CA, Fernandez JA: New insights into the circadian rhythm of acute myocardial infarction in subgroups. Chronobiol Int 2007, 24(1):129-141. 
31. Cannon CP, McCabe CH, Stone PH, Schactman M, Thompson B, Theroux P, Gibson RS, Feldman T, Kleiman NS, Tofler GH, et al: Circadian variation in the onset of unstable angina and non-Q-wave acute myocardial infarction (the TIMI III Registry and TIMI IIIB). Am J Cardiol 1997, 79(3):253-258.

32. Björk J, Forberg JL, Ohlsson M, Edenbrandt L, Öhlin H, Ekelund U: A simple statistical model for prediction of acute coronary syndrome in chest pain patients in the emergency department. BMC Med Inform Decis Mak 2006, 6(6):28.

doi:10.1186/1756-0500-5-420

Cite this article as: Ekelund et al:: Likelihood of acute coronary

syndrome in emergency department chest pain patients varies with time of presentation. BMC Research Notes 2012 5:420.

\section{Submit your next manuscript to BioMed Central and take full advantage of:}

- Convenient online submission

- Thorough peer review

- No space constraints or color figure charges

- Immediate publication on acceptance

- Inclusion in PubMed, CAS, Scopus and Google Scholar

- Research which is freely available for redistribution 